\title{
SHOCKED MOLECULAR GAS IN THE SUPERNOVA REMNANT HB 21
}

\author{
Bon-Chul Koo \\ Astronomy Program, SEES, Seoul National University, Seoul 151-742, Korea; \\ koo@astrohi.snu.ac.kr \\ Jeonghee Rho \& William T. Reach \\ Infrared Processing and Analysis Center, California Institute of Technology, Pasadena, CA 91125 \\ JaeHoon Jung \\ Korea Astronomy Observatory, Daeduk, Korea \\ Jeffrey, G. Mangum \\ NRAO, Tucson, AZ 85721-0665
}

\begin{abstract}
We have carried out ${ }^{12} \mathrm{CO} \mathrm{J}=2-1$ line observations of the supernova remnant (SNR) HB 21 in order to search for evidence of interaction with molecular clouds. We mapped the eastern half $\left(80^{\prime} \times 110^{\prime}\right)$ of the SNR almost completely. Molecular gas appears to be distributed mainly along the boundary of the SNR, but the overall distribution has little correlation either with the distortion of the SNR boundary or with the distribution of radio brightness. Along the eastern boundary, where the SNR was considered to be interacting with molecular clouds in previous studies, we have not found any strong evidence for the interaction. Instead we detected broad (20-40 $\left.\mathrm{km} \mathrm{s}^{-1}\right)$ CO emission lines in the northern and southern parts of the SNR. In the northern area, the broad-line emitting cloud is composed of a small $\left(\sim 2^{\prime}\right.$ or $0.5 \mathrm{pc}$ ), very bright, U-shaped part and several clumps scattered around it. There is a significant enhancement of radio emission with flat $(-0.28 \pm 0.17)$ spectral index possibly associated with this cloud. In the southern area, the broad-line emitting cloud is filamentary and appears to form an elongated loop of $\sim 30^{\prime}$ in extent. Small $\left(\lesssim 1^{\prime} .2\right.$ or $0.3 \mathrm{pc}$ ), bright clumps are seen along the filamentary structure. We have obtained sensitive $\mathrm{J}=1-0$ and $\mathrm{J}=2-1$ spectra of ${ }^{12} \mathrm{CO}$ and ${ }^{13} \mathrm{CO}$ molecules toward several peak positions. The intensity of ${ }^{12} \mathrm{CO} \mathrm{J}=2-1$ emission is low $\left(T_{m b}<7 \mathrm{~K}\right)$ and the ratio of ${ }^{12} \mathrm{CO} \mathrm{J}=2-1$ to $\mathrm{J}=1-0$ integrated intensities is high (1.6-2.3), which suggests that the emission is from warm, dense, and clumpy gas. We have applied an LVG analysis to derive their physical parameters. The detected broad CO lines are believed to be emitted from the fast-moving molecular gas swept-up by the SNR shock. The small ( $\lesssim 20 \mathrm{~km} \mathrm{~s}^{-1}$ ) shock velocity suggests that the shock is a non-dissociating C-shock. We discuss the correlation of the shocked molecular gas with the previously detected, shocked atomic gas and the associated infrared emission.
\end{abstract}


Subject headings: ISM: individual (HB 21) — ISM: molecules — radio lines — supernova remnants

\section{INTRODUCTION}

The number of supernova remnants (SNRs) with convincing evidence for interaction with ambient molecular clouds has increased considerably in recent years. The evidence ranges from a simple morphological relation to the detection of broad and/or shock-excited emission lines from various molecules. Although circumstantial evidence could be very suggestive, it is the molecular

lines from the shocked gas that are essential for understanding the physical and chemical processes associated with the molecular shock. In this regard, there are still only a few SNRs adequate for the study of molecular cloud-shock interaction; perhaps W28 (Arikawa et al. 1999), W44 (Seta et al. 1998), W51C (Koo \& Moon 1997), 3C391 (Reach \& Rho 1996, 1999), and the classical source IC 443 (DeNoyer 1979; Tauber et al. 1994 and references therein).

In this paper, we report the discovery of broad emission lines from the shocked $\mathrm{CO}$ gas in HB 21. HB 21 (G89.0+4.7) is one of those SNRs with mixed morphology, e.g., shell-like in radio and center-filled in the X-ray (Rho \& Petre 1998), where the center-filled, thermal X-ray emission is suggested to be due to interaction with molecular clouds. It has a nearly complete radio-continuum shell with an angular extent of $\sim 120^{\prime} \times 90^{\prime}$ (Hill 1974; Tatematsu et al. 1990, hereafter T90). The shell is elongated along the northwest-southeast direction. The brightness distribution of the shell is not uniform, but enhanced in scattered areas. Particularly noticeable features are the $\mathrm{V}$-shaped northern boundary, a $\sim 30^{\prime}$-sized loop structure in the south central area, and the one in the central region of the eastern boundary (see Fig. 1). Optical nebulosity associated with HB 21 has not been detected in $\mathrm{H} \alpha$ or [SII] plates (van den Bergh 1978). X-ray emission from HB 21 was detected by Leahy (1987) and studied in detail by Leahy \& Aschenbach (1996). The distance to the SNR is uncertain. We adopt $0.8 \mathrm{kpc}$ following T90, which is the distance to the Cyg OB 7 complex (Humphreys 1978).

HB 21 has been a suspect for interaction with molecular clouds based on its radio appearance and the ambient molecular clouds (Erkes \& Dickel 1969; Huang \& Thaddeus 1986; T90). Erkes \& Dickel (1969) suggested that the distorted boundaries with enhanced radio brightness might be the places where the SNR is interacting with dense ambient gas. Huang \& Thaddeus (1986) found that the giant molecular cloud associated with Cyg OB 7 appears to be partially surrounding HB 21 (see also Dobashi et al. 1994). T90 obtained a higher-resolution (2.'7) CO map of the eastern part of the SNR and found that the eastern boundary of the SNR appeared to be in contact with molecular and atomic clouds. They also made a coarsely-sampled map of the regions with enhanced radio emission and detected molecular clumps. But no direct evidence for the shocked molecular gas has been detected for HB 21. The search for $\mathrm{OH}$ masers, which are known 
to be an indicator for the interaction between a SNR and molecular cloud, gave negative results too (Frail et al. 1996). On the other hand, Koo \& Heiles (1991) detected shocked H I gas moving at 40 to $120 \mathrm{~km} \mathrm{~s}^{-1}$ in the southern part of the SNR, although the limited angular resolution $\left(36^{\prime}\right)$ hindered any detailed study of the shocked gas.

We detected broad $\mathrm{CO}$ emission lines in the northern and southern parts of HB 21, but not in the eastern part. We have found no evidence suggesting that the molecular clouds in the eastern part are interacting with the SNR, although they appear to be located along the boundary of the SNR on the sky. We summarize the observations and the results of molecular line observations in $\S 2$ and $\S 3$, respectively. In $\S 4$. we discuss the implications of our results on the interaction between the SNR and molecular clouds. $\S 5$ summarizes the main results of our paper.

\section{OBSERVATIONS}

${ }^{12} \mathrm{CO} \mathrm{J}=2-1$ line observations were carried out using the $12 \mathrm{~m}$ telescope of the National Radio Astronomy Observatory 1 at Kitt Peak in 1999 June and 2000 January. The FWHM of the telescope at $230 \mathrm{GHz}$ was $27^{\prime \prime}$. We mapped the eastern half $\left(80^{\prime} \times 110^{\prime}\right)$ of the SNR almost completely using the OTF (On-The-Fly) observing technique. We used two 256 channel filter banks; one with $500 \mathrm{kHz}$, and the other with $1 \mathrm{MHz}$ resolution. We split each filter bank into two sections and observed two linear polarizations simultaneously. The velocity resolution and coverage of the $500 \mathrm{kHz}$ filter bank were $0.65 \mathrm{~km} \mathrm{~s}^{-1}$ and $83 \mathrm{~km} \mathrm{~s}^{-1}$, while those of the $1 \mathrm{MHz}$ filter bank were two times greater. Typical system temperatures were 350-450 K. During the observing run in January 2000, we had some trouble because of telluric CO $\mathrm{J}=2-1$ emission, which appeared at $v_{\mathrm{LSR}}=4-5 \mathrm{~km} \mathrm{~s}^{-1}$. The emission was not cancelled out completely by usual position-switching observation and produced a hill-and-valley feature in spectra, the strength of which depends on elevation. We were able to avoid the contamination from telluric $\mathrm{CO}$ emission by averaging out the contaminated velocity channels because the telluric $\mathrm{CO}$ emission line is narrow $\left(0.8 \mathrm{~km} \mathrm{~s}^{-1}\right)$ and the absolute strength of the 'hill' and 'valley' features are equal.

We also obtained sensitive spectra of ${ }^{13} \mathrm{CO} \mathrm{J}=2-1,{ }^{12} \mathrm{CO} \mathrm{J}=1-0$ and $\mathrm{J}=2-1$ lines toward several peak positions. For $\mathrm{CO} J=1-0$ observations, we used 1 and $2 \mathrm{MHz}$ filter banks, so that they have the same velocity resolution and coverage with those of $\mathrm{CO} \mathrm{J}=2-1$ line observations. We also used the millimeter autocorrelator (MAC) for the ${ }^{12} \mathrm{CO}$ observations, which provided high resolution $\left(0.25 \mathrm{~km} \mathrm{~s}^{-1}\right.$ after smoothing) spectra. We have converted the observed temperatures $\left(T_{R}^{*}\right)$ to the main-beam brightness temperatures $\left(T_{m b}\right)$ using the corrected main-beam efficiency provided by the NRAO.

Additional observations of ${ }^{12} \mathrm{CO}$ and ${ }^{13} \mathrm{CO} \mathrm{J}=1-0$ lines were performed using the Taeduk

\footnotetext{
${ }^{1}$ The National Radio Astronomy Observatory is operated by Associated Universities, Inc., under contract with the National Science Foundation.
} 
Radio Astronomy Observatory (TRAO) $13.7 \mathrm{~m}$ telescope $\left(\mathrm{HPBW}=49^{\prime \prime}\right.$ at $115 \mathrm{GHz}$ ) in 2000

January and March. An SIS receiver equipped with a quasi-optical sideband filter was used along with a $250 \mathrm{kHz}, 256$-channel filter bank. The main beam efficiency was 0.41 at $115 \mathrm{GHz}$ (Roh \& Jung 1999), and the pointing accuracy was better than $10^{\prime \prime}$. Typical system temperatures were about $750 \mathrm{~K}$ at $115 \mathrm{GHz}$ and $450 \mathrm{~K}$ at $110 \mathrm{GHz}$.

\section{CO RESULTS}

\subsection{Overall Distribution and Clouds in Eastern Area}

Figure 1 shows the distribution of the integrated intensity of $\mathrm{CO} \mathrm{J}=2-1$ emission. The velocity range is between $v_{\mathrm{LSR}}=+3.9 \mathrm{~km} \mathrm{~s}^{-1}$ and $-17.5 \mathrm{~km} \mathrm{~s}^{-1}$, which covers most of the emission. The overlaied contour map shows the $1420 \mathrm{MHz}$ brightness distribution of HB 21 (T90). The overall distribution of CO gas in Figure 1 is not very different from the low-resolution map of T90. But Figure 1 shows much detailed structure because of its high resolution, high sensitivity, and complete sampling. Figure 1 immediately shows that molecular gas is distributed mainly along the boundary of the SNR. (We observed the central area in Figure 1, which had not been covered in our $\mathrm{CO} \mathrm{J}=2-1$ observations, in $\mathrm{CO} \mathrm{J}=1-0$ line emission, and have detected only several small $\left[\sim 1^{\prime}\right]$ clumps other than some faint extension associated with the clouds in southern and eastern parts of the remnant.) But the overall distribution has little correlation either with the distortion of SNR boundaries or with the distribution of radio brightness (cf. § 3.2). For the purpose of discussion, we divide the remnant into three areas (Fig. 2): (1) eastern area (RA> $20^{\mathrm{h}}$ $47^{\mathrm{m}}$ ) where three relatively large $\left(\gtrsim 15^{\prime}\right)$ clouds and several filamentary clouds are present, $(2)$ northern area centered at $\left(20^{\mathrm{h}} 46^{\mathrm{m}}, 51^{\circ} 00^{\prime}\right)$, where a small $\left(\sim 2^{\prime}\right)$, very bright U-shaped cloud is noticeable, and $(3)$ southern area centered at $\left(20^{\mathrm{h}} 44^{\mathrm{m}}, 49^{\circ} 50^{\prime}\right)$, where clumpy and filamentary clouds with complicated structures are present. We detected broad (20-40 $\mathrm{km} \mathrm{s}^{-1}$ ) emission lines from the clouds in the northern and southern areas, which will be discussed in detail in the next sections. In the following, we summarize the results on the eastern area.

In the eastern area, there are three clouds centered at declinations $\delta \simeq 50^{\circ} 49^{\prime}, 50^{\circ} 15^{\prime}$, and $\lesssim 49^{\circ} 50^{\prime}$. We call these three clouds by clouds A, B, and C following T90 (see Fig. 2 for the location of these clouds). Figure 3 shows the channel maps of the eastern area. The velocity ranges of the channel maps were chosen to show the essential features clearly. Cloud A appears at $v_{\mathrm{LSR}}=+9$ to $-6 \mathrm{~km} \mathrm{~s}^{-1}$ and is composed of two velocity components centered at $+6 \mathrm{~km} \mathrm{~s}^{-1}$ and $-2 \mathrm{~km} \mathrm{~s}^{-1}$, respectively. The former component $\left(6 \mathrm{~km} \mathrm{~s}^{-1}\right)$, which is seen in Figure $3 \mathrm{a}$, is extended and the emission peaks at the southern part $\left(\delta \simeq 50^{\circ} 45^{\prime}\right)$ of the cloud, while the latter component $\left(-2 \mathrm{~km} \mathrm{~s}^{-1}\right)$, which is seen in Figures $3 \mathrm{~b}$ and $3 \mathrm{c}$, is spatially confined and comprises the northern part of the cloud. Their maximum brightnesses are $T_{m b, \max }=11 \mathrm{~K}$ and $7 \mathrm{~K}$,

respectively. Cloud B appears at $v_{\mathrm{LSR}}=+1$ to $-9 \mathrm{~km} \mathrm{~s}^{-1}$ and is seen in Figures $3 \mathrm{c}$ and $3 \mathrm{~d}$. The south central part of the cloud, e.g., the region between $\delta=50^{\circ} 10^{\prime}-17^{\prime}$, is bright and appears to 
be connected to cloud C. Cloud $\mathrm{C}$ has two components at very different velocities, e.g., one at +2 to $-11 \mathrm{~km} \mathrm{~s}^{-1}$, which is seen in Figures $3 \mathrm{c}$ and $3 \mathrm{~d}$, and the other at +17 to $+10 \mathrm{~km} \mathrm{~s}^{-1}$, which is not shown in Figure 3 but has a distribution similar to the other velocity component. According to the result of T90, cloud $\mathrm{C}$ extends to $\delta \simeq 49^{\circ} 40^{\prime}$.

An interesting feature in Figure 3 is the semi-circular loop that appears above cloud B in Figure $3 \mathrm{~b}$. The ratio of the minor, which is along the NS direction, to the major axis is 0.8 . If it is at $0.8 \mathrm{kpc}$, the linear size of the semimajor axis would be $R_{s}=3 \mathrm{pc}$. The velocity increases systematically from both ends to the northern top of the loop, which is consistent with an expanding loop. The top portion is redshifted with respect to the both ends by $\simeq 3 \mathrm{~km} \mathrm{~s}^{-1}$. If we assume that the ellipticity is due to projection, then the expansion velocity would be $v_{\exp } \simeq 5 \mathrm{~km} \mathrm{~s}^{-1}$, so that the dynamical age of the ring is probably shorter than $R_{s} / v_{\text {exp }} \simeq 6 \times 10^{5}$ yrs. This is much greater than the age $\left(3 \times 10^{4}\right.$ yrs, Koo \& Heiles 1991 , scaled to $0.8 \mathrm{kpc}$ adopted in this paper) of HB 21 and, therefore, the loop might not be associated with HB 21. We suspect that the loop is originated from some energetic phenomena in cloud B. A faint 'V-shaped' structure that connects cloud B and the ends of the loop in Figure 3b seems to indicate the association of the two.

\subsection{Northern U-Shaped Cloud}

The cloud in the northern area is composed of a small $\left(\sim 2^{\prime}\right)$, very bright, U-shaped cloud and several clumps scattered around it (Fig. 1; see also Fig. 9 for enlarged view). Figure 4 shows its velocity structure. There are several points to be made from Figure 4: First the U-shaped cloud is composed of several clumps, whose central velocities shift systematically from +3 to $-6 \mathrm{~km} \mathrm{~s}^{-1}$ as we move from NE to NW along the structure. The integrated intensity attains a maximum at $\left(20^{\mathrm{h}}\right.$ $46^{\mathrm{m}} 03^{\mathrm{s}} .2,51^{\circ} 00^{\prime} 00^{\prime \prime}$ ), which we call HB21:BML-N1 (Broad Molecular Line - Northern Position 1 ), or simply N1. Second, there are several other clumps in the field. These clumps, except the one near the southeastern corner at $v_{\mathrm{LSR}}=-13 \mathrm{~km} \mathrm{~s}^{-1}$, appear over a wide $\left(>10 \mathrm{~km} \mathrm{~s}^{-1}\right)$ velocity range. Among them, the one at $\left(20^{\mathrm{h}} 45^{\mathrm{m}} 55 .^{\mathrm{s}} 0,51^{\circ} 03^{\prime} 30^{\prime \prime}\right)$, which we call HB21:BML-N2 (or $\mathrm{N} 2$ ), appears over the widest $\left(\simeq 30 \mathrm{~km} \mathrm{~s}^{-1}\right)$ velocity interval. Third, there is a diffuse emission at $+2 \mathrm{~km} \mathrm{~s}^{-1}$ to the $\mathrm{NE}$ of the cloud. Its line is narrow $\left(2-3 \mathrm{~km} \mathrm{~s}^{-1}\right)$ and it is part of a large $\left(\sim 20^{\prime}\right)$ cloud that appears to be connected to cloud A. We consider that the clumps aligned along the NE-SW direction in Figure 4 are associated and call them cloud N, i.e., cloud $\mathrm{N}$ does not include the diffuse emission in the northeastern area and the clump in the southeastern corner. (cf. T90 detected in this area only the diffuse molecular gas at $v_{\mathrm{LSR}}=1$ to $6 \mathrm{~km} \mathrm{~s}^{-1}$ and called it cloud D.)

As can be expected from Figure 4, most clumps in cloud $\mathrm{N}$ have broad emission lines. As an example, we show the spectra of N1 and N2 in the top frames in Figure 5, where we see that the spectrum of N1 is box-shaped and its full width (at zero intensity) is $30 \mathrm{~km} / \mathrm{s}$, while that of N2 is asymmetric and extends from -21 to $+11 \mathrm{~km} / \mathrm{s}$. For comparison, the spectrum of the diffuse, extended structure in the northeastern part of this area has narrow $\left(2-3 \mathrm{~km} \mathrm{~s}^{-1}\right)$ emission lines 
centered at $+2 \mathrm{~km} \mathrm{~s}^{-1}$, a sample of which is shown in the right bottom frame in Figure 5 .

We have obtained sensitive $\mathrm{J}=1-0$ and $\mathrm{J}=2-1$ spectra of ${ }^{12} \mathrm{CO}$ and ${ }^{13} \mathrm{CO}$ molecules at the two peak positions, N1 and N2, and Figure 5 shows the spectra. The molecule and transition are marked in each spectrum. The second spectrum from the top is ${ }^{12} \mathrm{CO} \mathrm{J}=2-1$ emission convolved to the $\mathrm{J}=1-0$ beam size $\left(55^{\prime \prime}\right)$ to be compared with the $\mathrm{J}=1-0$ spectra. The difference between the top and convolved spectra indicates that some velocity components, e.g., the narrow component centered at $+4 \mathrm{~km} \mathrm{~s}^{-1}$ of $\mathrm{N} 1$ and the broad component at $-8 \mathrm{~km} \mathrm{~s}^{-1}$ of $\mathrm{N} 2$, are confined to small areas. By comparing the $\mathrm{J}=1-0$ and the convolved $\mathrm{J}=2-1$ spectra, we notice that the ratio of $\mathrm{J}=2-1$ to $\mathrm{J}=1-0$ intensities is high and that it varies over the profile: For N1, the ratio is between 1.2 and 2.0 in the central parts of the spectrum, while it increases at the wings, e.g., $\sim 5$ at $+5 \mathrm{~km} \mathrm{~s}^{-1}$ and $-13 \mathrm{~km} \mathrm{~s}^{-1}$. For N2, the ratio varies between 0.8 and 2.8 , and it is higher between $-11 \mathrm{~km} \mathrm{~s}^{-1}$ and $0 \mathrm{~km} \mathrm{~s}^{-1}$. The ratios of the ${ }^{12} \mathrm{CO} \mathrm{J}=2-1$ and $\mathrm{J}=1-0$ integrated intensities ${ }^{12} R_{2-1 / 1-0}$ are 1.6 and 1.7 for $\mathrm{N} 1$ and $\mathrm{N} 2$, respectively. The ${ }^{13} \mathrm{CO} \mathrm{J}=2-1$ line is clearly detected toward N1, while it is marginally detected toward N2. For N1, the line has double peaks centered at +1 and $-9 \mathrm{~km} \mathrm{~s}^{-1}$, while the ${ }^{12} \mathrm{CO} \mathrm{J}=2-1$ line profile toward N1 is composed of several narrow peaks. The narrow peaks might indicate that the emission is from several, unresolved subclumps. Presumably, the ${ }^{13} \mathrm{CO}$ emission might be from these subclumps too, which is not apparent in the profile in Figure 5 because of low signal-to-noise ratio and low velocity resolution. (Note that the velocity resolutions of the ${ }^{12} \mathrm{CO} \mathrm{J}=2-1$ and ${ }^{13} \mathrm{CO} \mathrm{J}=2-1$ lines are 0.25 and $0.68 \mathrm{~km} \mathrm{~s}^{-1}$, respectively.) The ratios of ${ }^{12} \mathrm{CO} \mathrm{J}=2-1$ to ${ }^{13} \mathrm{CO} \mathrm{J}=2-1$ integrated line intensities ${ }^{12 / 13} R_{2-1}$ are $20 \pm 3$ and $40 \pm 14$ for N1 and N2, respectively. (The errors are statistical errors.) Table 1 summarizes the line parameters of the peak positions, i.e., their coordinate, velocity range $\left(v_{\min }\right.$, $\left.v_{\max }\right)$, CO $\mathrm{J}=2-1$ peak brightness temperature $T_{m b \text {,max }},{ }^{12} R_{2-1 / 1-0},{ }^{12 / 13} R_{2-1}$, and the ratio of ${ }^{12} \mathrm{CO} \mathrm{J}=1-0$ to ${ }^{13} \mathrm{CO} \mathrm{J}=1-0$ integrated line intensities ${ }^{12 / 13} R_{1-0}$. The detailed line diagnostics based on the observed line parameters in Table 1 is discussed in $\S 3.4$.

\subsection{Southern Filamentary Cloud}

In the southern part of the SNR, the emission is detected at $v_{\mathrm{LSR}}=-35$ to $+20 \mathrm{~km} \mathrm{~s}^{-1}$. The velocity structure is shown in Figure 6. At positive velocities, we see several clouds with narrow lines come and go, e.g., a diffuse cloud that extends $\sim 10^{\prime}$ along the NS direction centered at $\left(20^{\mathrm{h}}\right.$ $\left.44^{\mathrm{m}} .5,50^{\circ} 00^{\prime}\right)$ between $v_{\mathrm{LSR}}=0$ and $+5 \mathrm{~km} \mathrm{~s}^{-1}$. The $\mathrm{CO}$ distribution at negative velocities is fairly complicated: the distribution is filamentary, and small ( $\lesssim 1^{\prime} .2$ or $0.3 \mathrm{pc}$ ), bright clumps are seen along the filamentary structure. The filamentary structure, which we call cloud $\mathrm{S}$, appears to form a loop of $\sim 30^{\prime} \times 10^{\prime}$ in extent, elongated along the NS direction. The eastern part of the loop is particularly clumpy and has a semicircular shape (see the channel map centered at $\left.-10.1 \mathrm{~km} \mathrm{~s}^{-1}\right)$.

The clumps generally have broad ( $\gtrsim 10 \mathrm{~km} \mathrm{~s}^{-1}$ ) lines. Among them, three clumps marked by crosses in Figure 6 have broadest $\left(30-40 \mathrm{~km} \mathrm{~s}^{-1}\right)$ lines and we show the ${ }^{12} \mathrm{CO} \mathrm{J}=2-1$ and 
$\mathrm{J}=1-0$ spectra at their peak positions, which we call S1, S2, and S3 from east to west (see Fig. 2 ), in Figure 7. Again we show the convolved $\mathrm{J}=2-1$ spectra together, although the line shapes do not change significantly by convolution toward these peak positions. We have obtained some sensitive $\mathrm{J}=1-0$ and $\mathrm{J}=2-1$ spectra of ${ }^{12} \mathrm{CO}$ and ${ }^{13} \mathrm{CO}$ molecules at these peak positions, and the line parameters are listed in Table 1 . Note that ${ }^{12} R_{2-1 / 1-0}(=1.7-2.3)$ and ${ }^{12 / 13} R_{2-1}(=28 \pm 4$ toward S1) are similar to those of northern positions, while ${ }^{12 / 13} R_{1-0}$ 's, although they have large uncertainties, appear to be much greater than that of N1.

\subsection{Excitation Parameters of Broad Emission Lines}

The broad CO lines are presumably emitted from the shocked gas, where physical parameters vary greatly over a short distance scale. But still it would be worthwhile to estimate their excitation parameters based on elementary considerations. First, the observed ${ }^{12 / 13} R_{2-1}=20-40$ are significantly less than either the average ratio $(67.3 \pm 1.5$; Langer 1997$)$ of ${ }^{12} \mathrm{C} /{ }^{13} \mathrm{C}$ in the solar neighborhood or the terrestrial value (89), implying that the ${ }^{12} \mathrm{CO} \mathrm{J}=2-1$ lines are not optically thin. If we adopt ${ }^{12} \mathrm{C} /{ }^{13} \mathrm{C}=67$ and assume that the emission is thermalized, then the optical depth for the ${ }^{12} \mathrm{CO} \mathrm{J}=2-1$ line $\tau_{21}=1.1-3.4$. On the other hand, the ${ }^{12 / 13} R_{1-0}(\simeq 100 \pm 50)$ close to or greater than the terrestrial value imply that ${ }^{12} \mathrm{CO} \mathrm{J}=1-0$ lines are optically thin, except at $\mathrm{N} 1$ where $\mathrm{J}=1-0$ and $\mathrm{J}=2-1$ lines appear to have comparable optical depths. This, however, is not conclusive because of large uncertainties associated with ${ }^{12 / 13} R_{1-0}$. Second, the large values of ${ }^{12} R_{2-1 / 1-0}=1.6-2.3$ imply that the broad-line emitting region is warm and dense. For typical molecular clouds, where the $\mathrm{J}=2$ level is subthermally excited, the ratio is usually less than unity. For example, molecular clouds in the local arm exhibit ratios ranging from 0.53 (Taurus) to 0.75 (Orion A) (Sakamoto et al. 1994, 1997). Our spectra of ambient gas also show this, e.g., see the spectra toward S2 in Fig. 7 where the narrow component at $+2 \mathrm{~km} \mathrm{~s}^{-1}$ has ${ }^{12} R_{2-1 / 1-0}=0.72$. But the large ratio is common for the shocked molecular gas in SNRs (see $\S 4.1$ ). Third, the low $(<7 \mathrm{~K})$ brightness temperature of $\mathrm{J}=2-1$ lines, regardless of their moderate optical depths, imply that the emitting region must be clumpy, i.e., composed of subclumps, and the emission is beam-diluted. From the above considerations, we may conclude that the broad emission lines are from warm, dense clumps with significant column densities so that the $2-1$ lines are optically thick.

We have applied the large-velocity-gradient (LVG) model (Scoville \& Solomon 1974; Goldreich \& Kwan 1974) to our broad CO lines in order to derive their excitation parameters. The model assumes an uniform, spherical cloud with a constant velocity gradient $(v(r) \propto r)$. If the lines are emitted from the shocked region where temperature and density vary greatly, the resulting parameters may be considered as 'average' values. Since we have found that the emission is beam-diluted, we have used the line ratios, ${ }^{12} R_{2-1 / 1-0}$ and ${ }^{12 / 13} R_{2-1}$, instead of brightness temperatures to determine the excitation parameters. According to our LVG analysis, the observed ratios are possible for $T_{k} \geq 50 \mathrm{~K}$. Figure $8 \mathrm{a}$ shows the result of our model computations 
when $T_{k}=100 \mathrm{~K}$, where curves of constant ${ }^{12} R_{2-1 / 1-0}$ and constant ${ }^{12 / 13} R_{2-1}$ are drawn in $\left(X\left({ }^{12} \mathrm{CO}\right) /(d v / d r), n\left(\mathrm{H}_{2}\right)\right)$ plane. $X\left({ }^{12} \mathrm{CO}\right) /(d v / d r)$ is the fractional abundance of ${ }^{12} \mathrm{CO}$ relative to $\mathrm{H}_{2}\left(X\left({ }^{12} \mathrm{CO}\right)\right)$ per unit velocity gradient interval. The asterisks $\left(^{*}\right)$ mark the observed ratios toward the peak positions where both ratios are obtained, i.e., N1, N2, and S1. According to Figure $8 \mathrm{a}, n\left(\mathrm{H}_{2}\right)=(3-7) \times 10^{3} \mathrm{~cm}^{-3}$ and $X\left({ }^{12} \mathrm{CO}\right) /(d v / d r) \simeq(1-4) \times 10^{-6} \mathrm{pc}\left(\mathrm{km} \mathrm{s}^{-1}\right)^{-1}$ at the three peak positions. There are multiple choices for N1, e.g., the same ratios are obtainable when $n\left(\mathrm{H}_{2}\right)=3 \times 10^{5} \mathrm{~cm}^{-3}$ and $X\left({ }^{12} \mathrm{CO}\right) /(d v / d r)=4 \times 10^{-7} \mathrm{pc}\left(\mathrm{km} \mathrm{s}^{-1}\right)^{-1}$. We adopt the lower density because it is comparable to the densities in the other peak positions and because the density of $>10^{5} \mathrm{~cm}^{-3}$ appears to be too high for the $\mathrm{CO}$ emission to explore. If the temperature becomes higher, both $n\left(\mathrm{H}_{2}\right)$ and $X\left({ }^{12} \mathrm{CO}\right) /(d v / d r)$ need to be greater.

In Figure $8 \mathrm{~b}$, we plot the expected $\mathrm{CO} J=2-1$ radiation temperature $J_{\nu}\left(T_{b}\right) \equiv$ $\left(h \nu / k_{B}\right) /\left[\exp \left(h \nu / k_{B} T_{b}\right)-1\right]$ which is just the brightness temperature when $h \nu \ll k_{B} T_{b}$ and the expected ${ }^{12 / 13} R_{1-0}$ from the same LVG model. Note that the expected radiation temperatures are much greater than the observed main-beam brightness temperature. We have estimated beam filling factors of $(7.7-8.8) \times 10^{-2}$ from the ratio of these two brightnesses. We have estimated the CO column densities $\simeq(2.4-11) \times 10^{17} \mathrm{~cm}^{-2}$ at these peak positions by $n\left(\mathrm{H}_{2}\right)\left[X\left({ }^{12} \mathrm{CO}\right) /(d v / d r)\right] \Delta v$, where $\Delta v=14-18 \mathrm{~km} \mathrm{~s}^{-1}$ is the velocity width. The excitation

parameters derived from the LVG analysis are listed in Table 2 . Note that the ${ }^{12 / 13} R_{1-0}$ expected from the LVG model differ from the observed ones: At N1, the observed value is small by a factor of 2 , while, at $\mathrm{S} 1$, it is large by a factor of $2 \pm 1$. Considering the weakness of ${ }^{13} \mathrm{CO} \mathrm{J}=1-0$ lines and various uncertainties associated with different telescopes, however, it is not obvious if this difference is critical.

We have made a crude estimate of the mass of the broad-line clouds as follows. If the CO $\mathrm{J}=1-0$ line emission is optically thin, then $M\left(\mathrm{H}_{2}\right)$ can be obtained from the $\mathrm{CO} \mathrm{J}=1-0$ luminosity $L_{10}\left({ }^{12} \mathrm{CO}\right)$ by $M\left(\mathrm{H}_{2}\right)=L_{10}\left({ }^{12} \mathrm{CO}\right) m_{\mathrm{H}_{2}} /\left[h \nu_{10} A_{10} f_{J=1} X\left({ }^{12} \mathrm{CO}\right)\right]$ where $f_{J=1}$ is the fraction of $\mathrm{CO}$ molecules at $J=1$ level and the other coefficients have their usual meanings. In our case, $\mathrm{CO} J=1-0$ emission has less optical depth than the $\mathrm{J}=2-1$ emission, but is not very optically thin, so that the above formula might yield an underestimate. What we have is the luminosity of CO $\mathrm{J}=2-1$ emission $L_{21}\left({ }^{12} \mathrm{CO}\right)$, which has moderate optical depth. But, since ${ }^{12} R_{2-1 / 1-0}=1.6-2.3$ at the peak positions, we may obtain $L_{10}\left({ }^{12} \mathrm{CO}\right)$ by assuming that $L_{10}\left({ }^{12} \mathrm{CO}\right)=(1 / 2)\left(\nu_{10} / \nu_{21}\right){ }^{3} L_{21}\left({ }^{12} \mathrm{CO}\right)$ where $\nu_{10}$ and $\nu_{21}$ are CO $J=1-0$ and $J=2-1$ line frequencies respectively. Finally we assume $f_{J=1}=0.2$, which is a mean value of those $(0.15-0.26)$ at the three peak positions obtained from the LVG analysis. We have found that the $\mathrm{H}_{2}$ masses of clouds $\mathrm{N}$ and $\mathrm{S}$ are $\sim 8 M_{\odot}$ and $\sim 55 M_{\odot}$, respectively. The mass of the central U-shaped part of cloud $\mathrm{N}$ is $\sim 3 M_{\odot}$ while the masses of the small clumps in cloud $\mathrm{S}$ are $\sim 1 M_{\odot}$.

\section{INTERACTION BETWEEN HB 21 AND MOLECULAR CLOUDS}




\subsection{Evidence for the Interaction}

Broad CO emission lines with large ${ }^{12} R_{2-1 / 1-0}$ in clouds $\mathrm{N}$ and $\mathrm{S}$ strongly suggest that they are being shocked. The observed velocity width is as large as $\sim 40 \mathrm{~km} \mathrm{~s}^{-1}$. Note that, toward this direction $\left(\ell=89^{\circ}\right)$, the LSR velocity permitted by the Galactic rotation is $\lesssim 0 \mathrm{~km} \mathrm{~s}^{-1}$, so that it is not impossible for broad lines to be produced by molecular clouds accidentaly aligned along the line of sight. But it is highly improbable that such alignment (over a few kpcs) occurs in very small $\left(1-2^{\prime}\right)$ areas on the sky. We also searched for protostellar candidates around the broad line emitting regions using the Infrared Astronomical Satellite (IRAS) Point Source Catalog, because broad lines can be emitted from the high-velocity gas associated with protostellar object too. We did not find any suspicious sources except one, IRAS 20444+4954, which is located close to the S2 clump, i.e., at $\left(29^{\prime \prime} \pm 14^{\prime \prime}, 68^{\prime \prime} \pm 11^{\prime \prime}\right)$ from the peak position in Table 1 . The source has been detected in two IRAS wavebands, i.e., 60 and $100 \mu \mathrm{m}$, with flux densities of $F_{60 \mu \mathrm{m}}=0.99$ Jy and $F_{100 \mu \mathrm{m}}=12.97 \mathrm{Jy}$. We have found that the source is located within a small $\left(\sim 1^{\prime} \times 2^{\prime}\right)$, bright $\left(T_{m b} \simeq 6 \mathrm{~K}\right) \mathrm{CO} \mathrm{J}=2-1$ core at $v_{\mathrm{LSR}} \simeq 1 \mathrm{~km} \mathrm{~s}^{-1}$, so that it might be a young stellar object associated with the $\sim 10^{\prime}$-sized, diffuse cloud in the northern part of the $+2.9 \mathrm{~km} \mathrm{~s}^{-1}$ map in Figure 6, not with the S2 clump. Also the velocity of the S2 clump is similar to those of the other fast-moving clumps in this area, which suggests that they have the common origin. Therefore, the broad lines that we detected are almost certainly from the fast-moving molecular gas swept-up by the SNR shock in HB 21.

Another indication that the broad $\mathrm{CO}$ lines are from the shocked gas is their high

${ }^{12} R_{2-1 / 1-0}=1.6-2.3$. As we have shown in $\S 3.4$, the high ratio implies that the emitting gas is warm and dense, which might be manifestation of shock. Indeed high ${ }^{12} R_{2-1 / 1-0}$ is a common property of the broad lines from the shocked molecular gas in SNRs: All six SNRs that are known to have broad molecular emission lines, i.e., W28 (Arikawa et al. 1999), 3C391 (Reach \& Rho 1999), W44 (Seta et al. 1998), W51C (Koo \& Moon 1997), HB 21 (this paper), and IC 443 (e.g., van Dishoeck et al. 1993), have ratios greater than 1, which implies that the broad CO lines in these SNRs are all emitted from warm and dense, shocked gas. Meanwhile, the maximum brightness temperature $(<7 \mathrm{~K})$ of $\mathrm{CO} \mathrm{J}=2-1$ line in HB 21 is significantly less than those of other SNRs even if it was obtained with a higher spatial resolution (0.1 pc), e.g., it is $33 \mathrm{~K}$ for W28 (Reach \& Rho 2000b) and IC 443 (van Dishoeck et al. 1993) when observed with a resolution of 0.2 pc. The much smaller CO $\mathrm{J}=2-1$ brightness temperature with comparable ${ }^{12} R_{2-1 / 1-0}$ imply that either the shocked gas in HB 21 is composed of much smaller clumps or is less dense.

On a large scale, HB 21 appears to be in contact with a giant molecular cloud (GMC) along its eastern boundary (Huang \& Thaddeus 1986; Tatematsu et al. 1990). The GMC is $130 \mathrm{pc} \times 70 \mathrm{pc}$ in extent (Dobashi et al. 1994), and the structure that we call clouds A, B, and C defines the western boundary of the GMC. T90 inspected the correlation between these clouds and the SNR in detail, and concluded that cloud A might be interacting with the SNR because it is located where the radio continuum boundary of the SNR is distorted. On the other hand, they concluded that clouds $\mathrm{B}$ and $\mathrm{C}$ might not, because there is no indication of the interaction in the 
distribution of radio brightness. According to our high-resolution observations, however, there is little relationship between the boundaries of cloud A and the SNR. Instead, since the velocity of the ambient molecular gas around HB 21 might be negative (see $\S 4.3$ ), we consider that clouds B and $\mathrm{C}$ have a better chance of interaction. But we have detected broad CO lines toward none of these eastern clouds. Even if a SNR is interacting with a molecular cloud, the broad lines may be absent, however. 3C 391 (G31.9+0.0), which appears to be located at the edge of a large molecular cloud, for example, has no broad CO lines along the interface (Wilner, Reynolds, \& Moffett 1998; Reach \& Rho 1999). But strong [OI] $63 \mu \mathrm{m}$ emission has been detected near the interface, which indicates that the SNR is interacting with the molecular cloud (Reach \& Rho 1996). This would happen if the shock is dissociative and molecules have not reformed.

\subsection{Enhanced Radio Emission Possibly Associated with Northern U-Shaped Cloud}

Figure 9 shows an enlarged view of the northern area overlaied with a $325 \mathrm{MHz}$ radio continuum map of the SNR. Note that there is an enhanced radio continuum emission elongated along the NE-SW direction in the central area. Its peak position falls exactly inside the U-shaped part of cloud N. The positional coincidence suggests that the enhancement is possibly associated with cloud N. We have derived a spectral index $\left(f l u x \propto \nu^{\alpha}\right)$ of $-0.28 \pm 0.17$ for the radio emission associated with the U-shaped central part using the $325 \mathrm{MHz}$ and $1420 \mathrm{MHz}$ maps. The derived index has a large uncertainty because of the confusing "background" level, but it appears to be flatter than the mean spectral index $-0.4 \pm 0.03$ of HB 21 (Willis 1973). For cloud S, there is no obvious correlation between the $\mathrm{CO}$ emission and radio continuum brightness, although the radio continuum appears to be bright around the cloud in general.

It is not obvious, observationally or theoretically, what determines whether or not radio synchrotron emission becomes enhanced when SN shock hits a dense cloud. Observationally, we see very limited correlation between radio continuum brightness and shocked molecular gas in some SNRs, i.e., the shocked molecular gas is not usually associated with radio continuum enhancement and vice versa (see also Chevalier 1999). In IC 443, for example, shocked molecular

gas is distributed in a fragmentary, flattened, ring, which partly overlaps with the radio continuum shell (e.g., see Dickman et al. 1992 for the shocked molecular gas and Green 1986 for the radio continuum). But the radio continuum is brightest in the northeastern part of the shell where there is no shocked molecular gas, and the radio continuum is not particularly bright toward the shocked molecular gas, perhaps except around the southern part of the molecular ring (see next). In 3C 391, there is a shocked molecular clump in the southern part of the SNR, but there is only a faint, local radio continuum peak at $\sim 1.5 \mathrm{pc}$ apart from the shocked clump, whose association cannot be confirmed (Reach \& Rho 1999). In W28, on the other hand, there is a ridge of radio continuum emission associated with the shocked molecular gas (Arikawa et al. 1999). Theoretically, it could be either the dense cloud or the surrounding intercloud medium where synchrotron emission becomes enhanced. If the shock propagating through the dense cloud is 
radiative, there will be a large compression of cosmic rays and magnetic field, which would increase the synchrotron emissivity (van der Lann 1962; Blandford \& Cowie 1982). But this mechanism may not work because the molecular shocks in old SNRs are not ionizing shocks and, therefore, high energy particles may escape from the shocked region (Draine \& McKee 1993; Chevalier 1999). On the other hand, the shocked intercloud medium surrounding the cloud, particularly the medium behind the cloud, could have enhanced synchrotron emissivity because of the increased magnetic field strength there (e.g., Jones \& Kang 1993; Mac Low et al. 1994). In HB 21, the peak of the enhanced radio emission is located behind the shocked cloud (Fig. 8). It is also noteworthy that the cloud has a U-shape, which is similar to what we would expect when a small cloud is swept up by a strong shock (e.g., Klein, McKee, \& Colella 1994; Mac Low et al. 1994). These morphological characteristics seem to suggest that the enhanced emission is not physically associated with the shocked cloud but with the shocked surrounding intercloud medium.

In some respects, the region around cloud $\mathrm{N}$ in HB 21 is similar to the flat $(\alpha \sim-0.2)$ spectral region in IC 443, which is also located behind a shocked molecular cloud (Green 1986; Keohane et al. 1997). In IC 443, Keohane et al. (1997) found that the flat spectral region is particularly bright in hard X-rays, which are most likely due to synchrotron radiation. They concluded that the enhanced hard X-ray emission and the flat spectral index is due to shock acceleration of cosmic rays behind dense clouds. More observational study is certainly needed for HB 21 in order to reveal the relationship between the northern U-shaped cloud and the enhanced radio emission.

\subsection{Nature of the Shock and Shock Parameters}

Cold molecular gas around HB 21 in general has central velocities between $v_{\mathrm{LSR}} \simeq-8 \mathrm{~km} \mathrm{~s}$ and $+6 \mathrm{~km} \mathrm{~s}^{-1}$, which must be the velocity range of the preshock gas. In the areas where broad lines have been detected, there is diffuse molecular gas at positive $\left(0\right.$ to $\left.+5 \mathrm{~km} \mathrm{~s}^{-1}\right)$ velocities (see Figures 4 and 6). If this gas represents the preshock gas, then, since the broad lines are centered and spread out mostly at negative velocities, the gas should have been shocked and accelerated toward us systematically. If we take $+3 \mathrm{~km} \mathrm{~s}^{-1}$ as the velocity of the preshock gas and if we take either the central or peak positions of the broad lines as the systematic velocities of the shocked gas, then the line-of-sight velocities of the shock would be $\lesssim 10 \mathrm{~km} \mathrm{~s}^{-1}$ and $\lesssim 20 \mathrm{~km} \mathrm{~s}^{-1}$ for clouds $\mathrm{N}$ and $\mathrm{S}$, respectively. And, if we take $0.9 R_{s}$ and $0.75 R_{s}\left(R_{s}=\right.$ the radius of the $\left.\mathrm{SNR}\right)$ as their projected distances from the center of the SNR, then their deprojected velocities are $\lesssim 23 \mathrm{~km} \mathrm{~s}^{-1}$ and $\lesssim 30 \mathrm{~km} \mathrm{~s}^{-1}$, respectively. Such coasting clumps are indeed theoretically expected for the old SNRs such as HB 21: Molecular clumps swept up by a SNR blast wave are accelerated by the shock propagating into the clumps and also by the ram pressure of interclump gas (e.g. McKee 1988). The characteristic timescale for acceleration is $R / v_{s}$ where $R$ is the radius of the clump, which becomes $\sim 1 \times 10^{4}$ yrs $\left(<t_{\text {age }} \sim 3 \times 10^{4}\right.$ yrs $)$ for the clumps in HB 21 using $R \simeq 0.25$ pc and $v_{s} \simeq 25 \mathrm{~km} \mathrm{~s}^{-1}$. The wings of the broad lines may be attributed to the gas accelerated by shocks propagating from sides. But one difficulty in this scenario would be that there is no clear 
correlation in the distributions of the broad lines between the preshock and postshock gases. In the southern area, for example, the preshock gas $\left(2-3 \mathrm{~km} \mathrm{~s}^{-1}\right.$ component) is distributed in a filamentary cloud extended along the NS direction, while the broad-line emitting clumps are distributed over a much wider area to the SW of this cloud. Also it appears rather awkward that there are no broad lines centered near the velocity of the preshock gas. Alternatively, it could be the molecular gas at negative velocities that is associated with the SNR, and, in the northern and southern areas, most, if not all, of the ambient gas may have been shocked. In this picture, the shocked gas may be coasting too, but not necessarily at high speeds because the velocity of the preshock gas is somewhere within the velocity range of the broad lines, e.g., near the velocity of the peak. The shock velocity determined from the line width is $\lesssim 20 \mathrm{~km} \mathrm{~s}^{-1}$. Between the two possible interpretations, we prefer the latter because of the difficulty with the former mentioned above. But, since the difference in the shock velocities between the two interpretations is $\lesssim 10 \mathrm{~km} \mathrm{~s}^{-1}$, the following discussion remains valid basically even if the velocity of the preshock gas is 0 to $+5 \mathrm{~km} \mathrm{~s}^{-1}$. (The derived properties of the shocked gas in $\S 3.4$ should remain valid too in either case because the emission from the preshock gas might be in narrow lines and its contribution to the integrated intensity of broad lines is expected to be small.)

The observed shock velocity is $\lesssim 20 \mathrm{~km} \mathrm{~s}^{-1}$. This is less that the critical velocity for the dissociation of molecules, which is $25-50 \mathrm{~km} \mathrm{~s}^{-1}$ depending on preshock density and magnetic field strength (Hollenbach \& McKee 1980; Draine, Roberge, \& Dalgarno 1983). Hence, the shock might be a non-dissociating C-shock. The observed integrated intensity of the CO $\mathrm{J}=2-1$ emission is $(3-9) \times 10^{-7}$ ergs $\mathrm{cm}^{-2} \mathrm{~s}^{-1} \mathrm{sr}^{-1}$ at the peak positions. If we consider the beam dilution ( $\left.\S 3.4\right)$, the actual surface brightness may be greater by an order of magnitude, e.g., $(4-10) \times 10^{-6}$ ergs $\mathrm{cm}^{-2} \mathrm{~s}^{-1} \mathrm{sr}^{-1}$. This is much larger than the angle-averaged surface brightness predicted from shock model computations. Draine \& Roberge (1984), for example, computed surface brightnesses expected for steady-state C-shocks propagating through molecular gas with different preshock conditions. According to their result, the angle-averaged surface brightness of CO $\mathrm{J}=2-1$ emission varies from $1 \times 10^{-7}$ to $2 \times 10^{-6} \mathrm{ergs} \mathrm{cm}^{-2} \mathrm{~s}^{-1} \mathrm{sr}^{-1}$ for a $10-20 \mathrm{~km} \mathrm{~s}^{-1}$ shock propagating through a molecular cloud with $n\left(\mathrm{H}_{2}\right)=5 \times 10^{2}$ to $5 \times 10^{3} \mathrm{~cm}^{-3}$. Larger shock velocity does not raise the surface brightness while higher preshock density may yield $\leq 3 \times 10^{-6} \mathrm{ergs} \mathrm{cm}^{-2} \mathrm{~s}^{-1} \mathrm{sr}^{-1}$. The much higher surface brightness toward the peak positions would be possible if these are directions where we are observing the shock tangentially.

We want to briefly discuss the non-detection of $\mathrm{OH} 1720 \mathrm{MHz}$ masers in $\mathrm{HB} 21$, because such masers are known to indicate interaction of SNRs with molecular clouds (Frail et al. 1996). Firstly, the $\mathrm{OH}$ masers may require a very specific set of physical conditions that might not be realized in HB 21. According to Lockett, Gauthier, \& Elitzer (1999), the $1720 \mathrm{MHz}$ masers arise only in C-shocks when $T=50-125 \mathrm{~K}, n\left(\mathrm{H}_{2}\right) \sim 10^{5} \mathrm{~cm}^{-3}$, and $\mathrm{OH}$ column density of $10^{16}-10^{17} \mathrm{~cm}^{-2}$. According to our result in $\S 3.4$, the density of the shocked molecular gas in HB 21 appears to be much lower than required. Secondly, it is not impossible that the $\mathrm{OH}$ maser emission, even if present, had been missed in the survey by the Frail et al. (1996) who mapped the SNRs in 
rectangular grids with full-beam (or $2 \times$ full-beam) grid spacing. It would be worthwhile to search for $\mathrm{OH}$ masers toward the shocked $\mathrm{CO}$ gas in $\mathrm{HB} 21$.

Koo \& Heiles (1991) detected shocked H I gas associated with HB 21. The shocked H I gas moves at $v_{\mathrm{LSR}}=42-123 \mathrm{~km} \mathrm{~s}^{-1}$ and is confined to the southern part $\left(\delta=50^{\circ} 0^{\prime}-50^{\circ} 30^{\prime}\right)$ of the SNR. The highest velocity component coincides with cloud $\mathrm{S}$, although the angular resolution $\left(36^{\prime}\right)$ of the HI observation is too large for a detailed comparison. Koo \& Heiles (1991) assumed that the shocked H I gas represents a cap portion of a large expanding H I shell and derived a mean ambient $\mathrm{H}$ I density of $3.7 \mathrm{~cm}^{-3}$ (when scaled to $0.8 \mathrm{kpc}$ adopted in this paper). If the molecular shock has been driven by this H I shell, then we can roughly estimate the density of the shell as follows: We assume that the shocked molecular gas is confined to a thin slab and that the radiative $\mathrm{H} I$ shell has an uniform density of $\rho_{r s}$. Then it is straightforward to show that $\rho_{r s}$ is related to the density of the molecular cloud $\rho_{c}$ by $\rho_{r s} \simeq \rho_{c}\left(v / v_{r s}\right)^{2}$ (for $v_{r s} \gg v$ ) where $v_{r s}$ and $v$ are the velocities of radiative shell and shocked molecular slab, respectively (e.g., see Chevalier 1999). For HB 21, $v_{r s} \sim 130 \mathrm{~km} \mathrm{~s}^{-1}$ and $v \sim 20 \mathrm{~km} \mathrm{~s}^{-1}$. And if we take the $\mathrm{H}_{2}$ density of the cloud $n\left(\mathrm{H}_{2}\right) \sim 1 \times 10^{3} \mathrm{~cm}^{-3}$, the density of the $\mathrm{H}$ I shell would be $n_{r s}(\mathrm{H}) \sim 47 \mathrm{~cm}^{-3}$. By comparing with the mean ambient density $\left(3.7 \mathrm{~cm}^{-3}\right)$, this implies a compression factor $\beta \sim 13$ for the H I shell. Such moderate compression would be obtained if the ambient magnetic field strength tangential to the shell is $B_{0} \simeq 2\left(8 \pi \rho_{0} v_{r s}^{2}\right)^{1 / 2} /(3 \beta) \sim 10 \mu \mathrm{G}$ where $\rho_{0}$ is the density of the ambient medium. (The equation is obtained by assuming that ambient magnetic field is uniform and magnetic pressure dominates the pressure in the shell. See Chevalier 1974 for a discussion.) Alternatively, the fast-moving H I gas could be the gas originally associated with the molecular clouds, i.e., the atomic and molecular shocks may be produced when the SNR shock hits a large molecular cloud. In this case the fast-moving $\mathrm{H}$ I gas represents the swept-up interclump medium or H I envelope of molecular cloud. High-resolution H I observation is needed to reveal the relation between the shocked atomic and molecular gases.

\subsection{Infrared Emission from HB 21}

We used archival data from IRAS to search for infrared emission associated with the remnant. In his catalog of infrared emission from supernova remnants, Arendt (1989) called HB 21 a "probable" infrared source at 12 and $60 \mu \mathrm{m}$, with total fluxes of $180 \pm 60$ and $800 \pm 350 \mathrm{Jy}$, respectively. The main source of uncertainty is confusion with unrelated emission in the Galactic plane, which cannot be easily separated in infrared images. Using the IRAS Sky Survey Atlas (ISSA; Wheelock et al. 1993), we created an image covering the region around HB 21 at 60 and $100 \mu \mathrm{m}$. There is extensive emission to the south, east, and west of the remnant, but with no clear correlation with the radio or $\mathrm{CO}$ image. The region toward the center of the remnant is relatively fainter than these edges, but the northern part of the remnant is also faint. It is not possible to tell whether the emission is related to a partial shell around the remnant or just fluctuations in the background emission. 
To search for infrared emission associated with the remnant in more detail, we obtained a dedicated IRAS HIRES (Aumann, Fowler, \& Melnyk 1990) image for a $2^{\circ}$ field centered on HB 21. HB 21 is visible in the IRAS HIRES images at all four wavelengths $(12,25,60$, and 100 $\mu \mathrm{m})$. The $60 \mu \mathrm{m}$ image, where HB 21 is most prominent, is shown in Figure 10. Comparing the IRAS and CO images, it is evident that the southern filamentary cloud, cloud $\mathrm{S}$, is detected as a long arc, with very similar location, shape and width. The $60 \mu \mathrm{m}$ surface brightness of the filament is typically $7 \mathrm{MJy} \mathrm{sr}^{-1}$, and its structure is clumpy, like that of the CO emission. But the peaks of $\mathrm{CO}$ and infrared emission do not match in detail, suggesting that the $60 \mu \mathrm{m}$ emission does not arise from the exact same regions as the CO. In the northern area, there is also a good correspondence between the infrared and $\mathrm{CO}$ emission. The general correspondence in the south and north, and partial overlap in the east, show that many of the infrared features around the edge of HB 21 are related to the remnant, although the infrared emitting regions differ in detail from the $\mathrm{CO}$ emitting regions.

The nature of the infrared emission from HB 21 could be either dust grains surviving the shock, or from spectral lines from shock-excited gas, or both. We have estimated the mean surface brightness and color of the $\mathrm{CO}$ clouds by using several faint regions in the field as background. The results are summarized in Table 3 . The far-infrared color ratio, $I_{60} / I_{100} \simeq 0.20$, for clouds $\mathrm{A}$, $\mathrm{B}$, and $\mathrm{C}$ is almost identical to that of diffuse cirrus clouds in the solar neighborhood (Boulanger \& Pérault 1988). This suggests that the infrared emission from clouds A, B, and C is most likely due to dust heated at the surface of the molecular clouds by the interstellar radiation field; specifically, it suggests that the infrared emission is not related to shock fronts into the clouds. On the other hand, clouds $\mathrm{N}$ and $\mathrm{S}$ have a significantly higher color ratio $I_{60} / I_{100} \simeq 0.27$. This enhanced color, and the morphological correspondence with the broad molecular line emitting regions, suggests that the infrared emission from clouds $\mathrm{N}$ and $\mathrm{S}$ is due to shocks in propagating into the clouds. Conversely, the normal infrared color of clouds A, B, and C is consistent with their being due to ambient molecular clouds.

If the infrared emission from clouds $\mathrm{N}$ and $\mathrm{S}$ is due to dust, then the relatively higher $I_{60} / I_{100}$ could be due to smaller or warmer dust grains. For dust heated by the average interstellar radiation field in the solar neighborhood, about half of the emission at $60 \mu \mathrm{m}$ is thought to be due to small, transiently heated grains (Draine \& Anderson 1985; Désert, Boulanger, \& Puget 1990). Thus, if the enhanced $60 \mu \mathrm{m}$ emission is due to dust grains, then clouds $\mathrm{N}$ and $\mathrm{S}$ may contain a larger fraction of small grains. An enhanced abundance of small grains would be expected if a significant fraction of larger grains were shattered behind the shock front. Observations of local cirrus clouds with significant velocities revealed that $I_{60} / I_{100} \simeq 0.29$ is typical for clouds with $V_{L S R}>30 \mathrm{~km} \mathrm{~s}^{-1}$, suggesting that grain shattering was significant in the shocks that accelerated local clouds to intermediate velocities (Heiles, Reach, \& Koo 1988). Theoretically, significant shattering is not predicted for slow shocks such as inferred from the widths of the CO lines, but faster shocks through somewhat lower-density interclump gas, with $V_{s}>100 \mathrm{~km} \mathrm{~s}^{-1}$, could produce the enhanced $60 \mu \mathrm{m}$ emission (Jones, Tielens, \& Hollenbach 1996). 
Spectral lines could contribute significantly to the infrared emission from clouds N and S. The most important lines in the IRAS passbands, based on infrared spectra of similar supernova remnants (Oliva et al. 1999, Cesarsky et al. 1999, Reach \& Rho 2000a), are [O III] $88 \mu \mathrm{m}$ in IRAS band 4, [O I] $63 \mu \mathrm{m}$ in band 3, [Fe II] $26 \mu \mathrm{m}$ in band 2, and [Ne II] $12.8 \mu \mathrm{m}$ and $\mathrm{H}_{2}$ lines in band 1. If we were to interpret all of the IRAS emission from cloud $\mathrm{S}$ as due to the ionic lines listed above, then, using the system response and bandwidths (Beichman et al. 1988), we find that the brightest line would be [O III] $88 \mu \mathrm{m}$, with intensity $I=1.3 \times 10^{-3} \mathrm{erg} \mathrm{s}^{-1} \mathrm{~cm}^{-2} \mathrm{sr}^{-1}$. Relative to this line, the other bright lines would have ratios $\lambda \lambda 63 / 88=0.27, \lambda \lambda 26 / 88=0.13$, and $\lambda \lambda 12.8 / 88=0.26$. The implied brightness of the [O I] $63 \mu \mathrm{m}$ line can be easily produced by shocks with velocities $\sim 100 \mathrm{~km} \mathrm{~s}^{-1}$ into moderate-density $\left(10^{3} \mathrm{~cm}^{-3}\right.$ ) gas (Hollenbach \& McKee 1989); however, such shocks do not produce as much [O III] emission as observed, because the column density of highly-ionized gas is insufficient. Slower shocks into denser gas can also produce [O I] $63 \mu \mathrm{m}$ lines this bright (Draine, Roberge, \& Dalgarno 1983). However, slower (C-type) shocks produce essentially no ionic line emission - especially not an ion such as O III. Nor would the slow shocks destroy grains adequately to produce significant gas-phase Fe II. Therefore, if the infrared emission is from slow shocks, the IRAS $100 \mu \mathrm{m}$ band emission is from dust, and the IRAS $60 \mu \mathrm{m}$ band emission is from a mix of dust and the [O I] $63 \mu \mathrm{m}$ line. The nature of the IRAS 12 and 25 $\mu \mathrm{m}$ band emission is more difficult to constrain. If the infrared emission is from slower shocks, such as inferred from the CO observations, then there is likely a contribution from $\mathrm{H}_{2}$ lines. $\mathrm{H}_{2}$ rotational lines are the dominant coolant for a range of molecular shocks (Rho et al. 2000, Reach \& Rho 2000a). For now, it is not possible to clearly tell what fraction of the infrared emission is from gas or dust. The nature of the infrared emission from HB 21 (and other supernova remnants) can be determined in the future using spectroscopy or narrow-band imaging.

\section{SUMMARY}

We have mapped the eastern half $\left(80^{\prime} \times 110^{\prime}\right)$ of the SNR HB 21 in ${ }^{12} \mathrm{CO} \mathrm{J}=2-1$ line emission almost completely. Our map, which has been completely sampled with $27^{\prime \prime}$ resolution, shows the detailed structure of molecular clouds in this area. We have detected broad CO lines with large ${ }^{12} R_{2-1 / 1-0}$ in the northern and southern parts of $\mathrm{HB} 21$, which is direct evidence for the interaction between molecular clouds and the SNR. In the following, we summarize the main results of this paper:

(1) We detected shocked molecular clouds, clouds $\mathrm{N}$ and $\mathrm{S}$, with broad (20-40 $\left.\mathrm{km} \mathrm{s}^{-1}\right)$ CO lines in the northern and southern parts of the SNR. Cloud $\mathrm{N}$ is composed of a small $\left(\sim 2^{\prime}\right.$ or $\left.0.5 \mathrm{pc}\right)$, very bright, U-shaped, clumpy part and several clumps scattered around it. Cloud S is filamentary and appears to form an elongated loop of $\sim 30^{\prime}$ in extent. Small ( $\lesssim 1^{\prime} .2$ or $0.3 \mathrm{pc}$ ), bright clumps are seen along the filamentary structure. The $\mathrm{H}_{2}$ masses of clouds $\mathrm{N}$ and $\mathrm{S}$ are $\sim 8 M_{\odot}$ and $\sim 55 M_{\odot}$, respectively.

(2) We have obtained sensitive $\mathrm{J}=1-0$ and $\mathrm{J}=2-1$ spectra of ${ }^{12} \mathrm{CO}$ and ${ }^{13} \mathrm{CO}$ molecules toward 
several peak positions of clouds $\mathrm{N}$ and $\mathrm{S}$. They have ${ }^{12} R_{2-1 / 1-0}=1.6-2.3$ and ${ }^{12 / 13} R_{2-1}=20-40$ with $\mathrm{CO} \mathrm{J}=2-1$ main-beam brightness temperature less than $7 \mathrm{~K}$. According to our LVG analysis, $T_{k} \geq 50 \mathrm{~K}$, and, for $T_{k}=100 \mathrm{~K}, n\left(\mathrm{H}_{2}\right)=(3-7) \times 10^{3} \mathrm{~cm}^{-3}$ and $N(\mathrm{CO}) \simeq(2.4-11) \times 10^{17} \mathrm{~cm}^{-2}$. The emitting region appears to fill a small (0.077-0.088) fraction of the beam.

(3) There is an enhanced radio emission which attains a maximum exactly inside the central U-shaped part of cloud N. The emission has a spectral index $(-0.28 \pm 0.17)$ flatter than that of the whole remnant. The association of this emission with cloud $\mathrm{N}$ needs to be explored.

(4) Clouds N and S are visible in the IRAS HIRES images at all four wavelengths (12, 25, 60, and $100 \mu \mathrm{m})$. They have the far-infrared color ratio $I_{60} / I_{100} \simeq 0.27$, which is significantly greater than that (0.20) of the other clouds in this area. This enhanced color, and the morphological correspondence with the broad molecular line emitting regions, suggests that the infrared emission from clouds $\mathrm{N}$ and $\mathrm{S}$ is due to shocks in propagating into the clouds.

(5) Along the eastern boundary of the SNR, three relatively large ( $\gtrsim 15^{\prime}$ or $3.5 \mathrm{pc}$ ) clouds and several filamentary clouds are present. No broad CO emission or enhanced 60/100 $\mu$ m color were detected in any of these clouds, and there is little relationship between the boundaries of the clouds and the SNR. Therefore, there is no strong evidence for the interaction of the SNR with molecular clouds along the eastern boundary.

We thank Tom Landecker for kindly providing the radio images of HB 21. B.-C. K. was supported by the S.N.U. Research Fund (99-9-2-041) and in part by the BK21 Project of the Korean Government. 


\section{REFERENCES}

Arendt, R. G. 1989, ApJS, 70, 181

Arikawa, Y., Tatematsu, K., Sekimoto, Y., \& Takahashi, T. 1999, PASJ, 51, L7

Aumann, H. H., Fowler, J. W., \& Melnyk, M. 1990, AJ, 99, 1674

Beichman, C. A. et al. 1988, Infrared Astronomical Satellite (IRAS) Catalogs and Atlases: Volume 1. Explanatory Supplement, NASA RP-1190 (NASA: Washington, DC)

Blandford, R. D., \& Cowie, L. L. 1982, ApJ, 260, 625

Boulanger, F., \& Pérault, M. 1988, ApJ, 330, 964

Cesarsky, D., Cox, P., Pineau Des Forêts, G., van Dishoeck, E. F., Boulanger, F., \& Wright, C. M. 1999, A\& A, 348, 945

Chevalier, R. A. 1974, ApJ, 188, 501

- 1999, ApJ, 511, 798

DeNoyer, L. K. 1979, ApJL, 232, L165

Désert, F. X., Boulanger, F., \& Puget, J.-L. 1990, A\&A, 237, 215

Dickman, R. L., Snell, R. L., Ziurys, L. M., \& Huang, Y.-L. 1992, ApJ, 400, 203

Dobashi, K., Bernard, J.-P., Yonekura, Y., \& Fukui, Y. 1994, ApJS, 95, 419

Draine, B. T., \& Anderson, N. 1985, ApJ, 292, 494

Draine, B. T., \& McKee, C. F. 1993, ARAA, 31, 373

Draine, B. T., Roberge, W. G., \& Dalgarno, A. 1983, ApJ, 264, 485

Draine, B. T., \& Roberge, W. G. 1984, ApJ, 282, 491

Erkes, J. W., \& Dickel, J. R. 1969, AJ, 74, 840

Frail, D. A., Goss, W. M., Reynoso, E. M., Giacani, E. B., Green, A. J., \& Otrupcek, R. 1996, AJ, 111,1651

Goldreich, P., \& Kwan, J. 1974, ApJ, 189, 441

Green, D. A. 1986, MNRAS, 221, 473

Heiles, C., Reach, W. T., \& Koo, B.-C. 1988, ApJ, 332, 313

Hill, I. E. 1974, MNRAS, 169, 59 
Hollenbach, D., \& McKee, C. F. 1980, ApJL, 241, L47

— 1989, ApJ, 342, 306

Huang, Y.-L., \& Thaddeus, P. 1986, ApJ, 309, 804

Humphreys, R. M. 1978, ApJS, 38, 309

Jones, T. W., \& Kang, H. 1993, ApJ, 402, 560

Jones, A. P., Tielens, A. G. G. M., \& Hollenbach, D. J. 1996, ApJ, 469, 740

Keohane, J. W., Petre, R., Gotthelf, E. V., Ozaki, M., \& Koyama, K. 1997, ApJ, 484, 350

Klein, R. L., McKee, C. F., Colella, P. 1994, ApJ, 420, 213

Koo, B.-C., \& Heiles, C. 1991, ApJ, 382, 204

Koo, B.-C. \& Moon, D.-S. 1997, ApJ, 485, 263

Langer, W. D. 1997, in CO: Twenty-Five Years of Millimeter-Wave Spectroscopy, IAU Symp. 170, ed. Latter, W. B. et al. (Dordrecht: Kluwer), 98

Leahy, D. A. 1987, MNRAS, 228, 907

Leahy, D. A., \& Aschenbach, B. 1996, A\&A, 315, 260

Lockett, P., Gautheir, E., Elitzur, M. 1999, ApJ, 511, 235

Mac Low, M.-M., McKee, C. F., Klein, R. I., Stone, J. M., \& Norman, M. L. 1994, ApJ, 433, 757

McKee, C. F. 1988, in Supernova Remnants and the Interstellar Medium, Proceedings of IAU Colloquium No. 101, ed. R.S. Roger and T.L. Landecker (Cambridge: Cambridge), 473

Oliva, E., Moorwood, A. F. M., Drapatz, S., Lutz, D., \& Sturm, E. 1999, A\& A, 343, 943

Reach, W. T., \& Rho, J. 1996, A\&A, 315, L277

—. 1999, ApJ, 511, 836

—. 2000a, Aph0007148

—. 2000b, in preparation

Rengelink, R. B., Tang, Y., de Bruyn, A. G., Miley, G. K., Bremer, M. N., Röttgering, H. J. A., \& Bremer, M. A. R. 1997, A\&AS, 124, 259

Rho, J., \& Petre, R. 1998, ApJL, 503, L167

Rho, J., Jarrett, T., Cutri, R. M., \& Reach, W. T. 2000, ApJ, submitted 
Roh, D.-G., \& Jung, J. H. 1999, Publications of the Korean Astronomical Society, 14, 123

Sakamoto, S., Hasegawa, T., Handa, T., Hayashi, M., \& Oka, T. 1997, ApJ, 486, 276

Sakamoto, S., Hayashi, M., Hasegawa, T., Handa, T., \& Oka, T. 1994, ApJ, 425, 641

Scoville, N. Z., \& Solomon, P. M. 1974, ApJL, 187, L67

Seta, M., Hasegawa, T., Dame, T. M., Sakamoto, S., Oka, T., Handa, T., Hayashi, M., Morino, J., Sorai, K., \& Usuda, K. S. 1998, ApJ, 505, 286

Tatematsu, K., Fukui, Y., Landecker, T. L., \& Roger, R. S. 1990, A\&A, 237, 189 (T90)

Tauber, J. A., Snell, R. A., Dickman, R. L., \& Ziurys, L. M. 1994, ApJ, 421, 570

van den Bergh, S. 1978, ApJS, 38, 119

van der Laan, H. 1962, MNRAS, 124, 179

van Dishoeck, E. F., Jansen, D. J., \& Phillips, T. G. 1993, A\&A, 279, 541

Wheelock S. L., Gautier, T. N., Chillemi, J., Kester, D., McCallon, H., Oken, C., White, J., Gregorich, D., Boulanger, F., and J. Good 1994, IRAS Sky Survey Atlas: Explanatory Supplement. (JPL/Caltech: Pasadena)

Willis, A. G., 1973, A\&A, 26, 237

Wilner, D. J., Reynolds, S. P., \& Moffett, D. A. 1998, AJ, 115, 247 
Fig. 1. $-{ }^{12} \mathrm{CO} \mathrm{J=2-1}$ integrated intensity map of $\mathrm{HB} 21$. The velocity range is from $v_{\mathrm{LSR}}=$ $+3.9 \mathrm{~km} \mathrm{~s}^{-1}$ to $-17.5 \mathrm{~km} \mathrm{~s}^{-1}$, and the integrated intensity varies from 0 to $64 \mathrm{~K} \mathrm{~km} \mathrm{~s}^{-1}$. The overlaied contour map shows the $1420 \mathrm{MHz}$ brightness distribution of HB 21 obtained by T90 using the DRAO synthesis telescope.

Fig. 2.- A finding chart showing the positions of the sources described in the text. The outermost box represents the area of our $\mathrm{CO} \mathrm{J}=2-1$ line observation while the inner square boxes represent the areas shown in Figures 3, 4, and 6. Thin solid contours show the boundary of CO distribution while dashed contour shows the radio continuum boundary of the SNR HB 21.

Fig. 3.- Channel maps of the eastern area. Central velocities are marked on the top of each map. Velocity widths are $3.3 \mathrm{~km} \mathrm{~s}^{-1}, 3.9 \mathrm{~km} \mathrm{~s}^{-1}, 4.6 \mathrm{~km} \mathrm{~s}^{-1}$, and $5.2 \mathrm{~km} \mathrm{~s}^{-1}$ for maps a, b, $\mathrm{c}$, and d, respectively. Contour levels, which represent average brightness temperature in that velocity interval, are evenly spaced, with an interval of $1 \mathrm{~K}$, starting at $\bar{T}_{m b}=1 \mathrm{~K}$. Grey scale plot distinguishes hills from valleys.

Fig. 4.- Same as Figure 3, but for the northern area. The crosses mark the peak positions, i.e., $\mathrm{N} 1$ and $\mathrm{N} 2$ from east to west, where ${ }^{13} \mathrm{CO}$ lines have been obtained. Velocity width of each map is $3.3 \mathrm{~km} \mathrm{~s}^{-1}$. Contour levels are $\bar{T}_{m b}=0.5,1,2,3$, and $4 \mathrm{~K}$.

Fig. 5.- Sensitive CO spectra of the northern peak positions N1 and N2. The molecule and transition are marked in each spectrum. The second spectrum from the top is ${ }^{12} \mathrm{CO} \mathrm{J}=2-1$ spectrum convolved to the $\mathrm{J}=1-0$ beam size $\left(55^{\prime \prime}\right)$ to be compared with the $\mathrm{J}=1-0$ spectra. The ${ }^{12} \mathrm{CO} \mathrm{J}=1-0$ spectrum in the right bottom frame, which is toward the position $4^{\prime}$ away in RA from N1, is shown for comparison.

Fig. 6.- Channel maps of the southern area. The crosses mark the peak positions, i.e., S1, S2, and S3 from east to west, where ${ }^{13} \mathrm{CO}$ lines have been observed. Central velocities are marked on the top of each map. Velocity width of each map is $6.5 \mathrm{~km} \mathrm{~s}^{-1}$. Contour levels are $\bar{T}_{m b}=0.75,1.5$, $3,4.5,6$, and $7.5 \mathrm{~K}$.

Fig. 7.- Sensitive CO spectra of the southern peak positions S1, S2, and S3. The molecule and transition are marked in each spectrum. The second spectrum from the top is ${ }^{12} \mathrm{CO} \mathrm{J}=2-1$ spectrum convolved to the $\mathrm{J}=1-0$ beam size $\left(55^{\prime \prime}\right)$. Dotted lines in ${ }^{12} \mathrm{CO} \mathrm{J}=2-1$ spectra mark the velocity $\left(v_{\mathrm{LSR}}=4\right.$ to $\left.5 \mathrm{~km} \mathrm{~s}^{-1}\right)$ where the spectra are corrupted by telluric CO emission.

Fig. 8. - Result of our LVG model analysis for $T=100 \mathrm{~K}$. (a) Curves of constant ${ }^{12} R_{2-1 / 1-0}$ (solid line) and constant ${ }^{12 / 13} R_{2-1}$ (dotted line) are drawn. The asterisks $\left({ }^{*}\right)$ mark the observed ratios toward the peak positions where both ratios are obtained, i.e., N1, N2, and S1. (b) Curves of constant $\mathrm{CO} \mathrm{J}=2-1$ radiation temperature and constant ${ }^{12 / 13} R_{1-0}$ are drawn. The asterisks $\left(^{*}\right)$ mark $\left(n\left(\mathrm{H}_{2}\right), X\left({ }^{12} \mathrm{CO}\right) /(d v / d r)\right)$ pairs determined from (a), i.e., they do not represent the observed radiation temperature and ${ }^{12 / 13} R_{1-0}$. 
Fig. 9. - Enlarged view of the northern area. Grey-scale map shows the distribution of CO J=2-1 integrated intensity while contour map shows the distribution of $325 \mathrm{MHz}$ continuum brightness obtained from the Westerbork Northern Sky Survey (Rengelink et al. 1997). Note that the position of radio continuum peak coincides with broad-line emitting CO cloud. Contour levels are evenly spaced, with an interval of $0.01 \mathrm{~K}$, starting at $\bar{T}_{m b}=0.01 \mathrm{~K}$.

Fig. 10.- The $60 \mu \mathrm{m}$ image of HB 21. The CO distribution is shown in solid contours. 
Table 1. Observed Parameters at the Peak Positions with Broad Emission Lines

\begin{tabular}{ccccccc}
\hline \hline Name & $\begin{array}{c}\left(\alpha_{1950}, \delta_{1950}\right) \\
\left(\mathrm{h} \mathrm{m} \mathrm{s},{ }^{\prime}{ }^{\prime \prime}\right)\end{array}$ & $\begin{array}{c}\left(v_{\min }, v_{\max }\right) \\
\left(\mathrm{km} \mathrm{s}^{-1}\right)\end{array}$ & $\begin{array}{c}T_{\max } \\
(\mathrm{K})\end{array}$ & ${ }^{12} R_{2-1 / 1-0}$ & $12 / 13 R_{2-1}$ & $12 / 13 R_{1-0}$ \\
\hline HB21:BML-N1 & $(204603.2,510000)$ & $(-20,+10)$ & 3.6 & 1.6 & $20 \pm 3$ & $20 \pm 1$ \\
HB21:BML-N2 & $(204555.0,510330)$ & $(-21,+11)$ & 3.1 & 1.7 & $40 \pm 14$ & $\ldots$ \\
HB21:BML-S1 & $(204437.2,494710)$ & $(-22,+20)$ & 2.7 & 1.9 & $28 \pm 4$ & $96 \pm 50$ \\
HB21:BML-S2 & $(204431.0,495520)$ & $(-27,+0)$ & 2.9 & 1.7 & $\ldots$ & $104 \pm 43$ \\
HB21:BML-S3 & $(204245.2,495650)$ & $(-35,+1)$ & 6.9 & 2.3 & $\ldots$ & $103 \pm 43$ \\
\hline
\end{tabular}


Table 2. Physical Parameters Derived from LVG Analysis

\begin{tabular}{cccccc}
\hline \hline Name & $\begin{array}{c}n\left(\mathrm{H}_{2}\right) \\
\left(10^{3} \mathrm{~cm}^{-3}\right)\end{array}$ & $\begin{array}{c}X\left({ }^{12} \mathrm{CO}\right) /(d v / d r) \\
\left(10^{-6} \mathrm{pc} / \mathrm{km}^{-1} \mathrm{~s}\right)\end{array}$ & $\begin{array}{c}N(\mathrm{CO}) \\
\left(10^{17} \mathrm{~cm}^{-2}\right)\end{array}$ & $\begin{array}{c}\text { beam-filling } \\
\text { factor }\end{array}$ & $\begin{array}{c}12 / 13 R_{1-0} \\
\mathrm{~N} 1\end{array}$ \\
\hline $\mathrm{N} 2$ & 6.1 & 4.3 & 11 & 0.077 & 40 \\
$\mathrm{~S} 1$ & 3.1 & 1.4 & 2.4 & 0.088 & 57 \\
\hline
\end{tabular}


Table 3. Infrared Properties of Molecular Clouds

\begin{tabular}{cccccc}
\hline \hline $\begin{array}{c}\text { Cloud } \\
\text { Name }\end{array}$ & $\begin{array}{c}I_{12} \\
\left(\mathrm{MJy} \mathrm{sr}^{-1}\right)\end{array}$ & $\begin{array}{c}I_{25} \\
\left(\mathrm{MJy} \mathrm{sr}^{-1}\right)\end{array}$ & $\begin{array}{c}I_{60} \\
\left(\mathrm{MJy} \mathrm{sr}^{-1}\right)\end{array}$ & $\begin{array}{c}I_{100} \\
\left(\mathrm{MJy} \mathrm{sr}^{-1}\right)\end{array}$ & $I_{60} / I_{100}$ \\
\hline $\mathrm{A}$ & $1.1(0.2)$ & $1.1(0.1)$ & $4.2(0.2)$ & $20.8(2.3)$ & $0.20(0.03)$ \\
$\mathrm{B}$ & $1.7(0.1)$ & $1.8(0.1)$ & $5.9(1.1)$ & $29.5(2.4)$ & $0.20(0.04)$ \\
$\mathrm{C}$ & $1.7(0.1)$ & $1.7(0.1)$ & $8.0(1.1)$ & $38.0(2.4)$ & $0.21(0.03)$ \\
$\mathrm{N}$ & $1.0(0.2)$ & $0.8(0.1)$ & $4.8(0.2)$ & $17.0(2.3)$ & $0.28(0.04)$ \\
$\mathrm{S}$ & $1.2(0.1)$ & $1.2(0.1)$ & $6.8(1.1)$ & $26.3(2.4)$ & $0.26(0.05)$ \\
\hline
\end{tabular}

NOTE.-Numbers in parentheses are estimated $(1 \sigma)$ errors. 
This figure "f1.gif" is available in "gif" format from: http://arxiv.org/ps/astro-ph/0012341v1 
This figure "f2.gif" is available in "gif" format from: http://arxiv.org/ps/astro-ph/0012341v1 
This figure "f3.gif" is available in "gif" format from: http://arxiv.org/ps/astro-ph/0012341v1 
This figure "f4.gif" is available in "gif" format from: http://arxiv.org/ps/astro-ph/0012341v1 


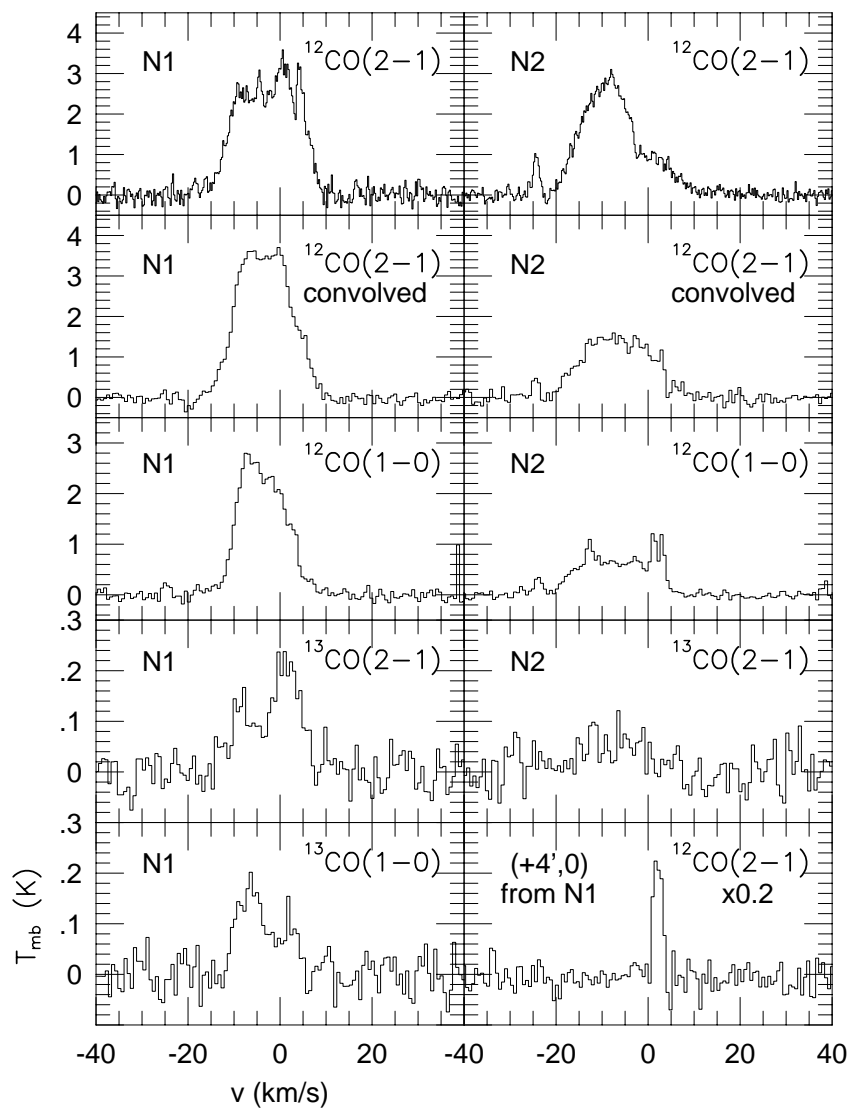


This figure "f6.gif" is available in "gif" format from: http://arxiv.org/ps/astro-ph/0012341v1 


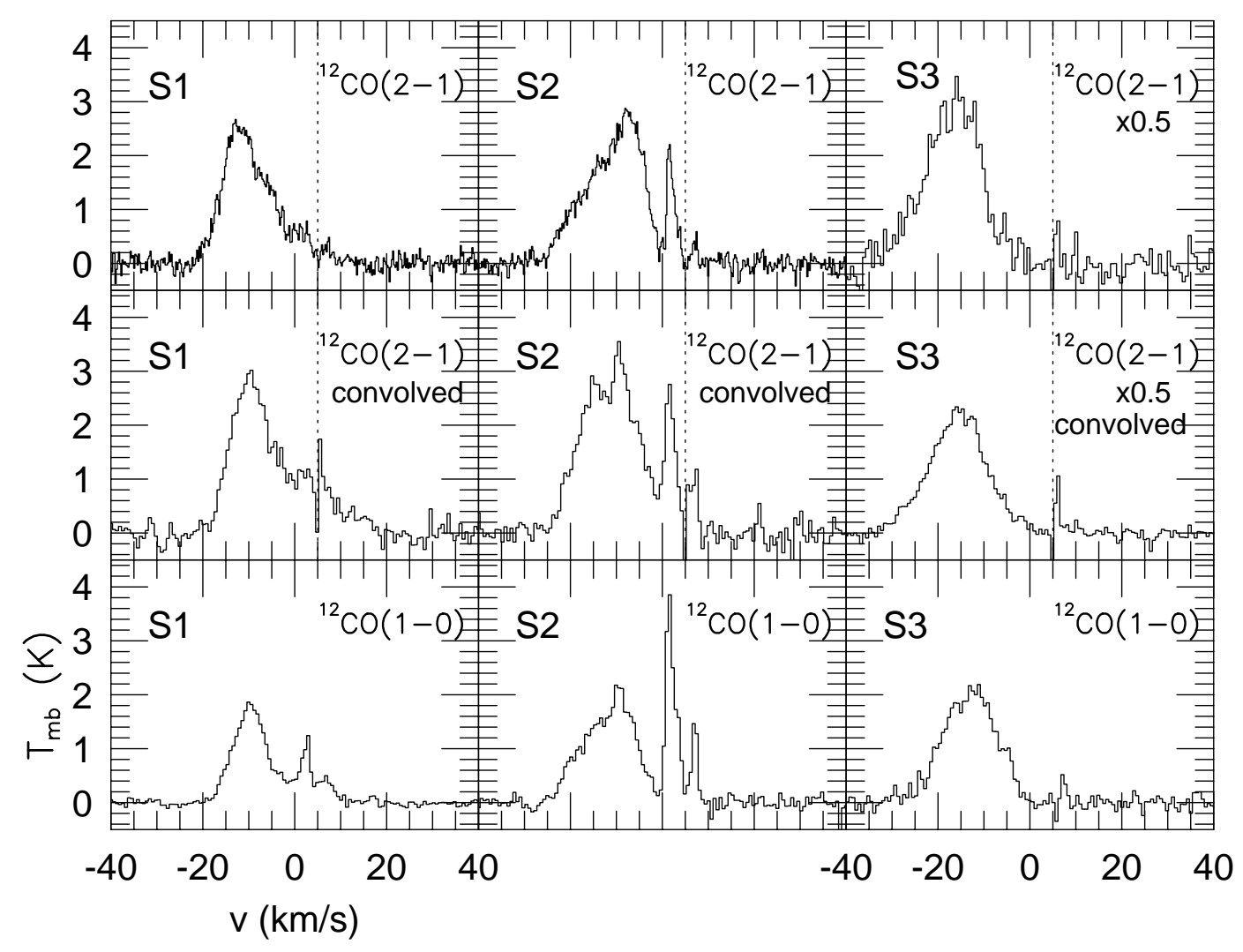




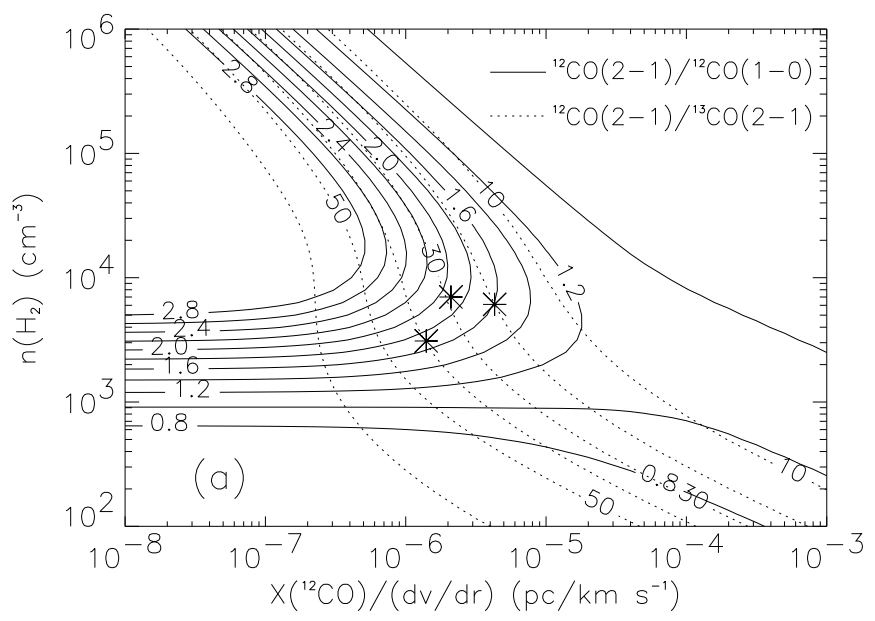




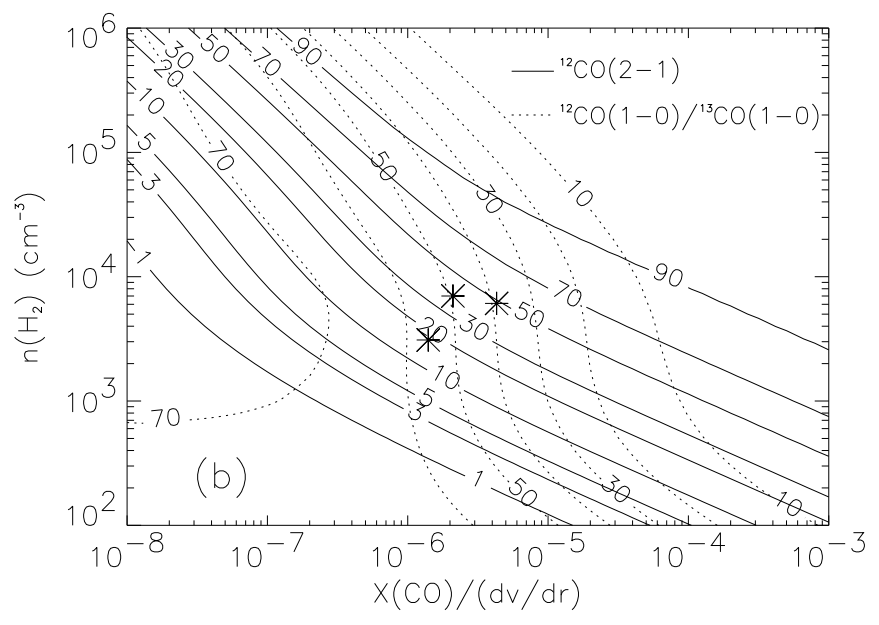




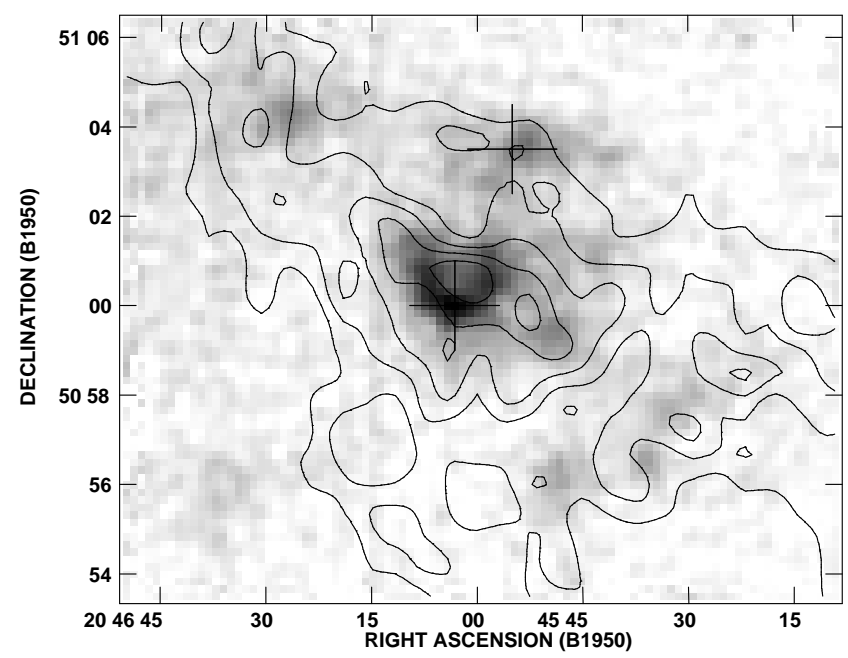


This figure "f10.gif" is available in "gif" format from: http://arxiv.org/ps/astro-ph/0012341v1 